\title{
CARACTERIZAÇÃO DE MÃES ADOLESCENTES E SEUS CONCEPTOS EM MUNICÍPIO DE PEQUENO PORTE, DE 1995 A 2009
}

Nayara Cristina Alves de Lima', Thaís Regina Ranussi², Mayckel da Silva Barreto ${ }^{3}$, Sonia Silva Marcon ${ }^{4}$

1Biomédica. Mestre em Biociências e Fisiopatologia. Instituto Maringá de Imagem. Maringá-PR-Brasil. 2Farmacêutica e Bioquímica. Mestre em Ciências da Saúde. Professora da Faculdade Dom Bosco de Cornélio Procópio. Bandeirantes-PR-Brasil. ${ }^{3}$ Enfermeiro. Mestre em Enfermagem. Professor da Faculdade de Filosofia, Ciências e Letras de Mandaguari. Maringá-PR-Brasil. ${ }^{4}$ Enfermeira. Doutora em Filosofia da Enfermagem. Professora da Universidade Estadual de Maringá. Maringá-PR-Brasil.

RESUMO: Estudo transversal que teve por objetivo caracterizar o perfil de mães adolescentes e comparar características sociais, obstétricas e do recém-nascido entre o grupo de adolescentes precoces (10 a 14 anos) e tardias (15 a 19 anos), no município de Bandeirantes, Paraná, de 1.995 a 2.009. As informações foram levantadas em junho de 2.012, no banco de dados do DATASUS. Os resultados evidenciaram que no período em estudo, ocorreram 8.364 partos, dos quais 1.833 (21,9\%) foram em adolescentes, sendo 73 (3,9\%) entre as precoces e 1.760 (96,1\%) entre as tardias. Esteve associada à gestação na adolescência precoce características como: menor escolaridade materna, ausência de companheiro, menor número de consultas de pré-natal e baixo peso ao nascer. Os achados reitera que piores condições sociodemográficas influenciam a ocorrência e o prognóstico da gravidez na adolescência, ressaltando a importância dos profissionais de saúde implementarem estratégias que diminuam sua (re)ocorrência.

DESCRITORES: Gravidez na adolescência; Perfil de saúde; Grupos de risco.

\section{CHARACTERIZATION OF ADOLESCENT MOTHERS AND THEIR NEWBORNS, IN A SMALL- SIZED CITY, FROM 1995 TO 2009}

\begin{abstract}
This is a cross-sectional study that aimed to characterize the profile of adolescent mothers, and to compare social and obstetric characteristics, as well as characteristics of the newborns, among a group of early adolescents (10-14 years old) and late adolescents (15-19 years old), in the city of Bandeirantes, Paraná, Brazil, from 1995 to 2009. Information was collected in June 2012, from the DATASUS database. The results evidenced that, in the period of the study, there were 8,364 deliveries, out of which 1,833 (21.9\%) were from adolescents - 73 $(3.9 \%)$ in early ones, and $1,760(96.1 \%)$ in late ones. Associated with pregnancy in early adolescence, there were characteristics like the mother's lower level of schooling, absence of a partner, smaller number of prenatal care visits, and low birth weight. The findings reiterate that worse socio-demographic conditions influence the occurrence and prognosis of adolescent pregnancy, reinforcing the importance health professionals have in the implementation of strategies that decrease its re (occurrence). DESCRIPTORS: Adolescent pregnancy; Health profile; Risk groups.
\end{abstract}

\section{CARACTERIZACIÓN DE MADRES ADOLESCENTES Y SUS CONCEPTOS EN UN PEQUEÑO MUNICIPIO, DE 1995 A 2009}

RESUMEN: Estudio transversal que tuvo por objetivo caracterizar el perfil de madres adolescentes y comparar características sociales, obstétricas y del recién nascido entre el grupo de adolescentes precoces (10 a 14 años) y tardías (15 a 19 años), en el municipio de Bandeirantes, Paraná, de 1995 a 2009. Las informaciones fueron obtenidas en junio de 2012, en el banco de datos del DATASUS. Los resultados evidenciaron que, en el periodo en estudio, ocurrieron 8.364 partos, de los cuales 1.833 $(21,9 \%)$ fueron en adolescentes, siendo $73(3,9 \%)$ entre las precoces y $1.760(96,1 \%)$ entre las tardías. Fueron asociadas a la gestación en la adolescencia precoz, características como: baja escolaridad materna, ausencia de compañero, poco número de consultas de prenatal y bajo peso al nacer. Los resultados apuntan que malas condiciones sociales y demográficas influencían la ocurrencia y el prognóstico de la gravidez en la adolescencia, destacando la importancia de la implantación de estratégias que ayuden a disminuir la (re)ocurrencia por parte de los profesionales de salud. DESCRIPTORES: Gravidez en la adolescencia; Perfil de salud; Grupos de riesgo.

*Artigo resultante da dissertação de Mestrado, Universidade Estadual de Maringá.

Recebido: $04 / 04 / 2013$ Finalizado: 06/02/2014

Autor correspondente:

Mayckel da Silva Barreto

Fundação Faculdade de Filosofia, Ciências e Letras de Mandaguari

Av. Lucílio de Held, 936 - 87033-230 - Maringá-PR-Brasil

E-mail: mayckelbar@gmail.com 


\section{INTRODUÇÃO}

A adolescência é compreendida entre os 10 e 19 anos, sendo uma fase do desenvolvimento humano essencial para que o indivíduo atinja sua maturidade biopsicossocial. Neste período, ocorre a busca pelo relacionamento interpessoal afetivo entre os jovens e a descoberta da sexualidade e de novas sensações corporais. Neste complexo contexto, de surpreendentes e emergentes transformações acontecem os primeiros contatos sexuais, e com isso, muitas vezes, os adolescentes se expõem às doenças sexualmente transmissíveis (DST) e a gravidez não planejada ${ }^{(1)}$.

A gestação na adolescência tem sido apontada como um "problema social". O aumento da gravidez nessa fase da vida traz preocupação, pois além do risco obstétrico ser maior, neste momento os jovens deveriam estar se preparando para a idade adulta, especialmente em relação aos estudos e melhor ingresso no mercado de trabalho(1). Ainda, o fato da maioria destes nascimentos ocorrer fora de uma relação conjugal estável tende a agravar a situação e por isso desperta a atenção da sociedade e dos serviços de saúde para o caso $^{(2)}$.

Diversos estudos, realizados em diferentes localidades, tem explorado o impacto da gravidez na adolescência sobre a mortalidade materna e neonatal, demonstrando haver maior incidência de complicações durante a gestação, o parto e o puerpério de adolescentes, tais como abortamento espontâneo, restrição de crescimento intrauterino, diabetes gestacional, pré-eclâmpsia, sofrimento fetal intraparto, parto prematuro, partos por cesárea com aumento de deiscência de suturas e dificuldade de amamentação ${ }^{(3-5)}$. Dentre as complicações referentes ao recém-nascido $(R N)$, observa-se aumento na incidência de desnutrição, hospitalização, maus tratos e acidentes domiciliares, que podem se estender durante toda a infância ${ }^{(6)}$.

Há, no entanto, poucas informações acerca do prognóstico obstétrico das adolescentes precoces (10 a 14 anos), quando comparado ao das adolescentes tardias (15 a 19 anos). Investigações a respeito de adolescentes grávidas, tipicamente, limitam sua população ao segundo grupo, enquanto que informações sobre mulheres mais jovens aparecem somente em estatísticas agregadas. Os poucos estudos que incluíram adolescentes mais jovens demonstram evidências inconclusivas em relação aos riscos associados à concepção nessa população. Isto pode refletir inconsistência na definição do limite para considerar a gestação na adolescente precoce ${ }^{(7)}$.

A gravidez na adolescência não planejada e a sua recorrência podem ser evitadas, por meio de acompanhamento multiprofissional qualificado e esclarecedor $^{(8)}$. Desta forma, torna-se necessário conhecer a magnitude do problema e o perfil das adolescentes e de seus filhos, buscando-se estruturar o conhecimento científico que subsidie a prestação da assistência ${ }^{(9)}$.

Outrossim, o reconhecimento dos fatores associados a problemática da gestação na adolescência e seus determinantes e agravos, é fundamental para direcionar o planejamento e a elaboração das políticas públicas sociais e de saúde, principalmente nas regiões onde o problema é considerado endêmico. Diante do exposto, o presente estudo teve como objetivos, caracterizar o perfil das mães adolescentes e comparar características sociais, obstétricas e do recém-nascido entre o grupo de adolescentes precoces (10 a 14 anos) e tardias (15 a 19 anos).

\section{MÉTODOS}

Estudo descritivo transversal realizado a partir do levantamento dos registros de todos os nascidos vivos de mães adolescentes, residentes no município de Bandeirantes, no período de 15 anos, compreendido entre primeiro de janeiro de 1.995 e 31 de dezembro de 2.009.

Bandeirantes é um município de pequeno porte, localizado na região norte do estado do Paraná, possui população de 32.184 habitantes e apresenta um índice de desenvolvimento humano (IDH) de 0,727, considerado médio. No setor público de saúde conta com oito Unidades Básicas de Saúde, seis equipes de Estratégias Saúde da Família (ESF), uma unidade de atendimento materno infantil e uma de atendimento para clínica geral.

Os dados foram coletados em junho de 2.012, por meio de consulta ao banco de dados eletrônico disponível no DATASUS - órgão do Ministério da Saúde responsável pelos sistemas informatizados. A fonte de informação foi a declaração de nascido vivo (DN), que é o formulário de coleta de dados do Sistema de Informação de Nascidos Vivos 
(SINASC) preenchido para todos os nascimentos ocorridos no país.

Após a consulta, os dados foram compilados em tabelas elaboradas pelos próprios pesquisadores e analisados estatisticamente no Excel for Windows ${ }^{\circledR}$ 2007. Para fins deste estudo, as mães adolescentes foram divididas, em dois grupos, de acordo com sua faixa etária (10 a 14 anos e 15 a 19 anos). As variáveis de interesse foram dicotomizadas e posteriormente divididas em: variáveis sócio demográficas (estado civil e anos de estudo), obstétricas (idade gestacional no momento do parto, tipo de gravidez, tipo de parto e número de consultas de pré-natal) e do RN (índice de Apgar no $1^{\circ}$ e $5^{\circ}$ minuto de vida e peso ao nascer). As informações que no banco de dados se apresentavam como ignoradas foram retiradas da análise.

Para diminuir a instabilidade das taxas e permitir um acompanhamento de como as variáveis em estudo se comportaram ao longo do tempo, os dados foram agrupados em três quinquênios (1995-1999, 2000-2004, 20052009) e se encontram distribuídos em tabelas de frequência absoluta e relativa. Para verificar a diferença entre os grupos avaliados foram utilizadas análises estatísticas, por meio do teste não-paramétricos qui-quadrado de Pearson. Houve considerável diferença numérica entre os grupos estudados. Desta forma, algumas variáveis apresentaram número de casos esperados menor que 05 e por isso, durante a análise dos dados também foi necessário empregar o teste de quiquadrado com correção de Yates. O nível de significância adotado foi $p<0,05$.

O estudo foi desenvolvido em consonância com a resolução 196/96 do Conselho Nacional de Saúde e aprovado pelo Comitê Permanente de Ética em Pesquisas com Seres Humanos da Universidade Estadual de Maringá, sob o número 005/2013, que dispensou o uso do consentimento livre esclarecido, uma vez que os dados foram obtidos, por meio de fontes secundárias.

\section{RESULTADOS}

De acordo com o DATASUS, de 1.995 a 2.009, no município em estudo, ocorreram 8.364 partos de nascidos vivos, dos quais 1.833 (21,9\%) foram em adolescentes, sendo 73 (3,9\%) entre as precoces e $1.760(96,1 \%)$ entre as tardias. O número de partos registrados foi maior no último quinquênio.

Observando as características sócio demográficas ao longo dos quinquênios nota-se que nos dois grupos de gestantes a porcentagem daquelas sem companheiro foi menor no segundo quinquênio seguida de uma considerável elevação no terceiro. Contudo, de modo geral, verifica-se que a maioria das adolescentes tardias possuía companheiro $(52,4 \%)$, enquanto que entre as precoces a proporção foi de $26,7 \%$, havendo diferença estatisticamente significativa entre os grupos (Tabelas 1, 2 e 3).

No que se refere à escolaridade materna observou-se que do primeiro para o segundo quinquênio houve considerável elevação no percentual de gestantes adolescentes com menos de oito anos de estudo, com tendência de estabilização das taxas no último período. No entanto, evidenciou-se também que $37,7 \%$ das adolescentes de 10 a 14 anos possuíam mais de oito anos de estudo, enquanto que para as de 15 a 19 anos esta proporção foi de $61 \%$, sendo constatada diferença estatisticamente significante entre os dois grupos.

Já em relação às variáveis obstétricas pode-se observar que apesar de a idade gestacional menor que 37 semanas por ocasião do nascimento e a gestação múltipla terem ocorrido com maior frequência entre as gestantes adolescentes precoces e o parto por cesárea entre as adolescentes tardias, estas variáveis não apresentaram diferenças estatisticamente significativas entre os dois grupos.

Quando se analisa a evolução no decorrer dos quinquênios da variável idade gestacional menor que 37 semanas no momento do parto, observa-se uma queda no número de gestantes adolescentes precoces que levaram a gestação até o termo. Já a variável tipo de parto mantevese estável no decorrer dos quinquênios entre as adolescentes precoces e tardias.

Pode-se evidenciar também que o menor número de consultas de pré-natal esteve associado com a gestação na adolescência precoce, sendo que pouco mais da metade (57,4\%) das mães adolescentes com idades entre 10 a 14 anos realizaram sete ou mais consultas de pré-natal, enquanto que entre as mais velhas este percentual chegou a 73,8\% (1.170 casos). 
Com relação às variáveis do $\mathrm{RN}$, pode-se observar que a maioria dos filhos de mães adolescentes precoces, apresentou boas condições de vitalidade (Apgar $>7)$ no primeiro $(87,5 \%)$ e no quinto minuto de vida (97,2\%). Quadro semelhante foi observado nos $\mathrm{RN}$ das mães de 15 a 19 anos onde a proporção de RN com score de Apgar superior a sete, no primeiro e quinto minuto de vida foram de $91,7 \%$ e $97,4 \%$ respectivamente, não havendo diferenças estatisticamente mensuráveis entre os dois grupos (Tabelas 4, 5 e 6).
No período em estudo, pode-se observar que houve aumento na proporção de crianças com baixo peso ao nascer entre as gestantes adolescentes precoces do primeiro para o segundo quinquênio, com tendência de estabilização do índice de casos do segundo para o terceiro quinquênio. Nota-se ainda que a maior parte dos RN de mães adolescentes tardias nasceu com o peso adequado (91,9\%), já entre as adolescentes precoces esta proporção foi inferior (82\%). Deste modo, evidenciou-se diferença estatisticamente significativa entre os grupos.

Tabela 1 - Distribuição das mães adolescentes, segundo variáveis sociais e obstétricas. Bandeirantes-PRBrasil, 1995-199

\begin{tabular}{|c|c|c|c|c|}
\hline \multirow{3}{*}{$\begin{array}{l}\text { Ano } \\
\text { Idade materna } \\
\text { Características } \\
\end{array}$} & \multicolumn{4}{|c|}{1995 - 1999} \\
\hline & \multicolumn{2}{|c|}{ 10-14 anos } & \multicolumn{2}{|c|}{ 15-19 anos } \\
\hline & $\mathrm{n}$ & $\%$ & $\mathrm{n}$ & $\%$ \\
\hline \multicolumn{5}{|l|}{ Estado civil } \\
\hline Com companheiro & 01 & 33,3 & 31 & 67,4 \\
\hline Sem companheiro & 02 & 66,7 & 15 & 32,6 \\
\hline \multicolumn{5}{|l|}{ Anos de estudo } \\
\hline$<8$ anos & 03 & 11,5 & 48 & 7,6 \\
\hline$\geq 8$ anos & 23 & 88,5 & 583 & 92,4 \\
\hline \multicolumn{5}{|l|}{ Idade gestacional } \\
\hline$<37$ semanas & 01 & 3,4 & 33 & 4,4 \\
\hline$\geq 37$ semanas & 28 & 96,5 & 702 & 95,6 \\
\hline \multicolumn{5}{|l|}{ Tipo de gestação } \\
\hline Única & 29 & 100 & 728 & 99 \\
\hline Múltipla & - & - & 07 & 1 \\
\hline \multicolumn{5}{|l|}{ Tipo de parto } \\
\hline Vaginal & 23 & 79,3 & 586 & 79,6 \\
\hline Cesárea & 06 & 20,7 & 150 & 20,4 \\
\hline \multicolumn{5}{|c|}{ Número de consultas de pré-natal } \\
\hline$<7$ & 06 & 24 & 79 & 14 \\
\hline$\geq 7$ & 19 & 76 & 483 & 86 \\
\hline
\end{tabular}


Tabela 2 - Distribuição das mães adolescentes, segundo variáveis sociais e obstétricas. Bandeirantes-PR-Brasil, 2000-2004

\begin{tabular}{|c|c|c|c|c|}
\hline \multirow{3}{*}{$\frac{\text { Ano }}{\text { Idade materna }}$} & \multicolumn{4}{|c|}{2000 - 2004} \\
\hline & \multicolumn{2}{|c|}{ 10-14 anos } & \multicolumn{2}{|c|}{ 15-19 anos } \\
\hline & $\mathrm{n}$ & $\%$ & $\mathrm{n}$ & $\%$ \\
\hline \multicolumn{5}{|l|}{ Estado civil } \\
\hline Com companheiro & 9 & 40,9 & 417 & 72,4 \\
\hline Sem companheiro & 13 & 59,1 & 159 & 27,6 \\
\hline \multicolumn{5}{|l|}{ Anos de estudo } \\
\hline$<8$ anos & 23 & 100 & 332 & 57,6 \\
\hline$\geq 8$ anos & - & - & 244 & 42,4 \\
\hline \multicolumn{5}{|l|}{ Idade gestacional } \\
\hline$<37$ semanas & 3 & 13 & 68 & 11,7 \\
\hline$\geq 37$ semanas & 20 & 87 & 509 & 88,3 \\
\hline \multicolumn{5}{|l|}{ Tipo de gestação } \\
\hline Única & 23 & 100 & 575 & 99,6 \\
\hline Múltipla & - & - & 2 & 0,4 \\
\hline \multicolumn{5}{|l|}{ Tipo de parto } \\
\hline Vaginal & 16 & 69,5 & 382 & 66,3 \\
\hline Cesárea & 7 & 30,5 & 194 & 33,7 \\
\hline \multicolumn{5}{|c|}{ Número de consultas de pré-natal } \\
\hline$<7$ & 11 & 47,8 & 197 & 34,1 \\
\hline$\geq 7$ & 12 & 52,2 & 380 & 65,9 \\
\hline
\end{tabular}

Tabela 3 - Distribuição das mães adolescentes, segundo variáveis sociais e obstétricas. Bandeirantes-PR-Brasil, 2005-2009

\begin{tabular}{|c|c|c|c|c|}
\hline \multirow{3}{*}{$\begin{array}{l}\text { Ano } \\
\text { Idade materna } \\
\text { Características }\end{array}$} & \multicolumn{4}{|c|}{$2005-2009$} \\
\hline & \multicolumn{2}{|c|}{ 10-14 anos } & \multicolumn{2}{|c|}{ 15-19 anos } \\
\hline & $\mathrm{n}$ & $\%$ & $\mathrm{n}$ & $\%$ \\
\hline \multicolumn{5}{|l|}{ Estado civil } \\
\hline Com companheiro & 2 & 10 & 112 & 25,1 \\
\hline Sem companheiro & 18 & 90 & 334 & 74,9 \\
\hline \multicolumn{5}{|l|}{ Anos de estudo } \\
\hline$<8$ anos & 17 & 85 & 265 & 59,4 \\
\hline$\geq 8$ anos & 3 & 15 & 181 & 40,6 \\
\hline \multicolumn{5}{|l|}{ Idade gestacional } \\
\hline$<37$ semanas & 2 & 10 & 20 & 4,4 \\
\hline$\geq 37$ semanas & 18 & 90 & 427 & 95,6 \\
\hline \multicolumn{5}{|l|}{ Tipo de gestação } \\
\hline Única & 20 & 95,2 & 445 & 99,5 \\
\hline Múltipla & 1 & 4,8 & 2 & 0,5 \\
\hline \multicolumn{5}{|l|}{ Tipo de parto } \\
\hline Vaginal & 14 & 70 & 294 & 65,7 \\
\hline Cesárea & 6 & 30 & 153 & 34,3 \\
\hline \multicolumn{5}{|c|}{ Número de consultas de pré-natal } \\
\hline$<7$ & 12 & 60 & 140 & 31,3 \\
\hline$\geq 7$ & 8 & 40 & 307 & 68,3 \\
\hline
\end{tabular}


Tabela 4 - Distribuição das características dos recém-nascidos de mães adolescentes. Bandeirantes-PR-Brasil, 1995-1999

\begin{tabular}{|c|c|c|c|c|}
\hline \multirow{3}{*}{$\begin{array}{l}\text { Ano } \\
\text { Idade materna } \\
\text { Características }\end{array}$} & \multicolumn{4}{|c|}{ 1995-1999 } \\
\hline & \multicolumn{2}{|c|}{ 10-14 anos } & \multicolumn{2}{|c|}{ 15-19 anos } \\
\hline & $\mathrm{n}$ & $\%$ & $\mathrm{n}$ & $\%$ \\
\hline \multicolumn{5}{|c|}{ Apagar no $1^{\circ}$ minuto } \\
\hline$\leq 7$ & 5 & 17,2 & 80 & 10 \\
\hline$>7$ & 24 & 82,8 & 715 & 90 \\
\hline \multicolumn{5}{|c|}{ Apagar no $5^{\circ}$ minuto } \\
\hline$\leq 7$ & 1 & 3,4 & 26 & 3,5 \\
\hline$>7$ & 28 & 96,6 & 709 & 96,5 \\
\hline \multicolumn{5}{|l|}{ Peso ao nascer } \\
\hline$<2500 \mathrm{~g}$ & 4 & 13,8 & 66 & 8,9 \\
\hline$\geq 2500 \mathrm{~g}$ & 25 & 86,2 & 669 & 91,1 \\
\hline
\end{tabular}

Tabela 5 - Distribuição das características dos recém-nascidos de mães adolescentes. Bandeirantes-PR-Brasil, 2000-2004

\begin{tabular}{|c|c|c|c|c|}
\hline \multirow{3}{*}{$\begin{array}{l}\text { Ano } \\
\text { Idade materna } \\
\text { Características }\end{array}$} & \multicolumn{4}{|c|}{ 2000-2004 } \\
\hline & \multicolumn{2}{|c|}{ 10-14 anos } & \multicolumn{2}{|c|}{ 15-19 anos } \\
\hline & $\mathrm{n}$ & $\%$ & $\mathrm{n}$ & $\%$ \\
\hline \multicolumn{5}{|c|}{ Apagar no $1^{\circ}$ minuto } \\
\hline$\leq 7$ & 4 & 17,4 & 42 & 7,2 \\
\hline$>7$ & 19 & 82,6 & 534 & 92,8 \\
\hline \multicolumn{5}{|c|}{ Apagar no $5^{\circ}$ 'minuto } \\
\hline$\leq 7$ & 1 & 4,3 & 15 & 2,5 \\
\hline$>7$ & 22 & 95,7 & 562 & 97,5 \\
\hline \multicolumn{5}{|l|}{ Peso ao nascer } \\
\hline$<2500 \mathrm{~g}$ & 5 & 21,7 & 42 & 7,2 \\
\hline$\geq 2500 \mathrm{~g}$ & 18 & 78,3 & 535 & 92,8 \\
\hline
\end{tabular}

Tabela 6 - Distribuição das características dos recém-nascidos de mães adolescentes. Bandeirantes-PR-Brasil, 2005-2009

\begin{tabular}{lcccc}
\hline Ano & \multicolumn{2}{c}{$\mathbf{2 0 0 5 - 2 0 0 9}$} & \\
\hline Idade materna & \multicolumn{2}{c}{$10-14$ anos } & $\mathrm{n}$ & 15-19 anos \\
\hline Características & $\mathrm{n}$ & $\%$ & 28 & 6,2 \\
\hline Apagar no $1^{\circ}$ minuto & & - & 419 & 93,8 \\
\hline$\leq 7$ & - & 100 & 6 & \\
\hline$>7$ & 20 & & 441 & 1,3 \\
\hline Apagar no $5^{\circ}$ minuto & & - & 98,7 \\
\hline$\leq 7$ & - & 100 & \\
\hline$>7$ & 20 & & 36 & 13,9 \\
\hline Peso ao nascer & & 20 & 411 & 86,1 \\
\hline$<2500 \mathrm{~g}$ & 4 & 80 & & \\
\hline$\geq 2500 \mathrm{~g}$ & 16 & &
\end{tabular}




\section{DISCUSSÃO}

Muitas pesquisas, sobre gestação na adolescência, tem sido realizadas no Brasil ${ }^{(7,10-13)}$ e no mundo ${ }^{(6,14-15)}$. No entanto, apesar desta temática ser constante nas discussões científicoacadêmicas, as adolescentes, muitas vezes, se deparam com a falta de serviços assistenciais qualificados, em decorrência do déficit de conhecimentos estruturados que auxiliem a prática dos profissionais nos serviços de saúde. Desta forma, não há um acompanhamento integral a esta parcela vulnerável da população, que objetive diminuir possíveis danos físicos e psicológicos ante a (re)vivência de uma gestação precoce.

Acredita-se que a elevação na taxa de natalidade observada no último quinquênio do período estudado, decorra da intensificação da cobrança dos gestores municipais sobre as notificações compulsórias, entre elas as de nascidos vivos, acarretando um incremento e maior rigor dispensado ao preenchimento das DN. Tal afirmação é corroborada pela diminuição no número de campos preenchidos como ignorados nas DN e nos bancos de dados do DATASUS no decorrer dos anos.

O índice de gestação na adolescência $(21,9 \%)$ e o de adolescentes precoces grávidas (3,9\%) verificado neste estudo é muito similar, ao encontrado em pesquisa análoga realizada no estado do Espírito Santo, a qual encontrou prevalência de gestação na adolescência de $20 \%$, sendo ainda que 3,89\% destas ocorreram em adolescentes com idade entre 10 e 14 anos $^{(11)}$.

Situação preocupante e que desperta a atenção é o fato de 73,3\% das mães adolescentes precoces não estarem em uma relação conjugal estável no momento do parto. Possivelmente, as jovens vivenciaram, dentre outros problemas, uma relação conflituosa com a família, o que tornou a gravidez na adolescência uma conjuntura ainda mais difícil de ser experenciada. Neste sentido, em muitos casos, a presença de um companheiro poderia representar fonte de apoio sócio-emocional para a adolescente grávida.

A situação conjugal das adolescentes é um dos fatores que influenciam no modo como as mesmas enfrentam o ciclo gravídico-puerperal. Sendo assim, os serviços de saúde devem estar atentos para esta informação, pois há evidências de que a presença do companheiro para a gestante adolescente desencadeia atitudes de auto-cuidado, como, por exemplo, maior adesão às consultas de pré-natal(6). Assim, é necessário que o enfermeiro da ESF conheça a situação conjugal das gestantes adolescentes de sua área adstrita, a fim de elaborar estratégias de intervenção que tragam os companheiros para o serviço de atenção à saúde das gestantes, auxiliando-as a aderir às atividades de prénatal e a vivenciar da melhor forma possível as dificuldades que ocorrem durante a gravidez.

A ausência do companheiro influencia ainda nas condições de nascimento dos filhos de mães adolescentes. Estudo de caso-controle, realizado no município de Rio Grande-RS, com 547 RN, dos quais 41,1\% eram filhos de mães adolescentes, demonstrou associação entre o baixo peso ao nascer e as mulheres sem companheiro. De acordo com os autores do referido estudo tal situação, pode relacionar-se a instabilidade emocional decorrente da falta de apoio psicológico do companheiro; renda familiar reduzida devido a uma única fonte; e extremos de idade, sobretudo, as mães adolescentes que mais comumente permanecem sem companheiro ${ }^{(16)}$.

A maior parte das gestantes adolescentes precoces $(62,3 \%)$ possuía menos de oito anos de estudo. Vale destacar, que a baixa escolaridade das mães adolescentes precoces, não constitui um indicativo social confiável, uma vez que a menor escolaridade pode ser explicada pelo fato de muitas delas ainda não terem idade suficiente para atingir os oito anos de estudo. Assim, o que se torna relevante para esta faixa etária é a investigação da continuidade dos estudos após a gravidez, pois a gestação na adolescência trás consequências importantes não apenas do ponto de vista biológico, mas também, quanto a aspectos sociais, já que entre as mães adolescentes é frequente o abandono ou adiamento dos estudos ${ }^{(11)}$.

O número de consultas de pré-natal apresentase como um qualificador da assistência à saúde oferecida à gestante. O Ministério da Saúde preconiza que sejam realizadas, no mínimo, sete consultas de pré-natal durante o transcorrer da gestação $^{(17)}$. A assistência pré-natal permite que muitas patologias maternas, que são capazes de afetar a saúde do concepto, possam ser tratadas ou controladas durante o período da gestação e do pós-parto, evitando, assim, efeitos danosos sobre a criança e a mãe. Além disso, a assistência 
pré-natal, quando bem realizada, pode contribuir para a diminuição da ocorrência de alguns problemas específicos do momento do parto ${ }^{(18)}$.

A proporção de gestantes que utilizou sete ou mais consultas de pré-natal foi menor entre as adolescentes precoces $(57,4 \%)$ do que entre as tardias (73,8\%). Esta situação decorre além da menor escolaridade e da falta de um companheiro, já citadas nesta reflexão, de outros fatores como, por exemplo, o início tardio do acompanhamento, visto que muitas vezes as adolescentes tentam esconder ou mesmo desconhecem seu estado gestacional; a qualidade do atendimento prestado pelos profissionais de saúde; e a imaturidade das adolescentes para o entendimento sobre a importância do acompanhamento de pré-natal, tanto para sua saúde quanto para a saúde da criança ${ }^{(19-20)}$.

Nesta perspectiva, estudo realizado em Rio Branco (AC) junto a 20 gestantes de baixo risco atendidas por consulta médica e de enfermagem na Atenção Primária, observou que as entrevistadas com idade superior a 25 anos reconheciam mais facilmente a relevância desse acompanhamento para a manutenção da sua saúde e a da criança, por ser um momento de realização de exames e de obtenção de informações e conhecimentos que evitam complicações na gestação e no parto e subsidiam a prestação dos cuidados pós-natais ${ }^{(8)}$.

O não comparecimento às consultas de pré-natal, por sua vez, está associado ao desenvolvimento de intercorrências durante o período gravídico, principalmente ao final da gestação, quando a maior frequência de visitas deva ocorrer, com vistas à avaliação do risco perinatal e das intercorrências clínico-obstétricas mais comuns no terceiro trimestre ${ }^{(17)}$.

Além do baixo número de gestantes adolescentes precoces que realizou mais de sete consultas de pré-natal, algo que pode ser apreendido como negativo foi o fato de que quando se analisa temporalmente a evolução desta variável, observa-se uma tendência de elevação no número de adolescentes que não aderiram às consultas de pré-natal. Tal situação merece atenção dos gestores e dos profissionais de saúde que atuam na Atenção Primária, a fim de que possam elaborar estratégias que captem precocemente e estimulem as gestantes a permanecerem no acompanhamento pré-natal.

Outra característica obstétrica importante é a idade gestacional por ocasião do parto, que neste estudo, apesar do baixo índice de prematuridade registrado, evidenciou uma discreta diferença entre os dois grupos, com predomínio de partos prematuros entre as adolescentes precoces, porém sem diferença estatisticamente mensurável.

Este fato também foi verificado em um estudo documental realizado em Fortaleza (CE) a partir da consulta ao prontuário de 2.058 parturientes adolescentes, o qual constatou que embora o total de partos prematuros tenha sido relativamente superior no grupo das adolescentes precoces, a diferença encontrada não foi estatisticamente significativa( ${ }^{(7)}$. Entretanto, outros autores reiteram que a taxa de parto prematuro é consideravelmente superior entre as adolescentes com idade entre 10 a 14 anos, e por consequência há maior possibilidade de complicações biopsicossociais ${ }^{(11)}$.

Ao ser analisada a evolução temporal do tipo de parto entre as adolescentes, fica evidente que as mesmas estão menos expostas às intervenções cirúrgicas. Deste modo, abrem-se possibilidades para a redução dos atuais níveis de partos cirúrgicos, o que constituiria objeto de investigações específicas. Talvez, a prática médica no sentido de realizar um maior número de partos por via vaginal entre as adolescentes, seja também um dos fatores que expliquem o porquê de não terem sido observados neste estudo elevados índices de baixo peso ao nascer e prematuridade.

Para se investigar possíveis diferenças no parâmetro de vitalidade dos RN de mães adolescentes precoces e tardias, analisou-se o score de Apgar no primeiro e no quinto minutos de vida, não sendo observadas diferenças significativas entre os dois grupos. Optou-se por analisar este parâmetro, pois os índices de Apgar no $1^{\circ}$ e ao $5^{\circ}$ minuto de vida representam a melhor escala de risco a respeito das condições clínicas do RN no momento do parto. No entanto, poucos são os estudos realizados com $\mathrm{RN}$ que trazem estatísticas acerca deste parâmetro(20).

Em um levantamento feito por meio do SINASC em Montes Claros (MG), que distinguiu adolescentes precoces (10 a 14 anos) das tardias (15 a 19 anos) e adultas (20 anos ou mais), os autores, de um modo geral, encontraram resultados significativamente piores nas adolescentes precoces, relacionados ao número de consultas pré-natal, maior prematuridade, maior risco para baixo peso ao nascer e Apgar menor que sete no 
quinto minuto de vida ${ }^{(19)}$.

Em relação ao baixo peso ao nascer, evidenciou-se, estatisticamente maior prevalência de nascidos vivos com menos de $2.500 \mathrm{~g}$ entre as mães adolescentes precoces. Sendo assim, é importante destacar que crianças com baixo peso ao nascer tem risco aumentado de morte no primeiro ano de vida, de desenvolverem enfermidades infecciosas e respiratórias, de evoluírem com atraso no desenvolvimento e de apresentarem, no futuro, doenças crônicas, como obesidade, dislipidemia e problemas cardíacos ${ }^{(20)}$.

De modo geral, os achados deste estudo puderam reiterar que piores condições sócio demográficos influenciam a ocorrência e o prognóstico da gravidez na adolescência, principalmente entre aquelas com menor idade, o que ressalta a importância dos profissionais de saúde implementarem estratégias que diminuam sua (re)ocorrência.

\section{CONSIDERAÇÕES FINAIS}

Os resultados do presente estudo apontam que adolescentes com idade entre 10 e 14 anos, quando comparadas com as adolescentes de 15 a 19 anos, apresentam determinadas características que influenciam negativamente no ciclo gravídico-puerperal, o que reforça a concepção de que a gravidez nesta faixa etária é de maior risco. Constatou-se que essas características podem influenciar nos cuidados com a saúde (número de consultas de pré-natal) e intervir nas condições de nascimento (baixo peso ao nascer) e até na forma como esta criança será criada (escolaridade materna, presença do pai). Daí a maior preocupação com o aumento da gravidez em idades mais precoces.

Acredita-se que essas especificidades dentro do grupo de adolescentes necessitam ser mais bem estudadas, para facilitar a compreensão destes fatores na gênese da gestação na adolescência e também devam ser consideradas na programação da oferta de serviços de atenção ao planejamento familiar, pré-natal e ao parto.

É mister conhecer o perfil da população materno-infantil de cada localidade, principalmente quando envolve adolescentes, pois, assim, pode-se atuar sobre as principais lacunas diagnosticadas. Estudos desta natureza servem de diretrizes para a elaboração de políticas sociais e de saúde, que objetivem a diminuição da ocorrência deste problema social.

Algo que merece destaque é a falta de uma importante informação no banco de dados do DATASUS, que é a recorrência da gestação em adolescentes. Sugere-se então, o acréscimo desta informação no banco de dados, uma vez que ela já é coletada durante o preenchimento da DN e pode fomentar novas análises e discussões acerca do tema.

\section{REFERÊNCIAS}

1. Spindola T, Silva LFF. Perfil epidemiológico de adolescentes atendidas no pré-natal de um Hospital Universitário. Esc. Anna Nery. 2009;13(1):99-107.

2. Pandin MFR, Silva RS, Chalem E, Mitsuhiro SS, Barros MM, Guinsburg R, et al. Brief report: A sociodemographic profile of multiparous teenage mothers. J adolesc. 2009;32(3):715-1.

3. Martins MG, Santos GHN, Sousa MS, Costa JFB, Simões VMF. Associação de gravidez na adolescência e prematuridade. Rev Bras Ginecol Obstet. 2011;33(11):354-60.

4. García H, Avendaño-Becerra NP, Islas-Rodríguez MT. Neonatal and maternal morbidity among adolescent and adult women. A comparative study. Rev. invest. clín. 2008;60(2):94-100.

5. Zeck W, Walcher W, Tamussino K, Lang U. Adolescent primiparas: changes in obstetrical risk between 1983-1987 and 1999-2005. J Obstet Gynaecol Res. 2008;34(2):195-8.

6. Nettle D, Coall DA, Dickins TE. Birthweight and paternal involvement predict early reproduction in British women: evidence from the National Child Development Study. Am J Hum Biol. 2010;22(2):172-7.

7. Magalhães MLC, Furtado FM, Nogueira MB, Carvalho FHC, Almeida FML, Mattar R, et al. Gestação na adolescência precoce e tardia - há diferença nos riscos obstétricos? Rev Bras Ginecol Obstet. 2006;28(8):446-52.

8. Pessoa IN, Menezes ED, Ferreira TF, Dotto LMG, Bessa LF. Percepção de puérperas sobre assistência de enfermagem na gravidez. Cienc. cuid. saude 2009;8(2):236-41.

9. Skinner SR, Smith J, Fenwick J, Hendriks J, Fyfe S, Kendall G. Pregnancy and protection: Per-ceptions, attitudes and experiences of Australian female adolescents. Wom Birth. 2009;22(2):50-6. 
10. Carniel EF, Zanolli ML, Almeida CAA, Morcillo AM. Características das mães adolescentes e de seus recém-nascidos e fatores de risco para a gravidez na adolescência em Campinas, SP, Brasil. Rev. Bras. Saude Mater. Infant. 2006;6(4):419-26.

11. Nader PRA, Cosme L. A. Parto prematuro de adolescentes: influência de fatores sóciode-mográficos e reprodutivos, Espírito Santo, 2007. Esc. Anna Nery. 2010;14(2):338-45.

12. Oliveira EFV, Gama SGN, Silva CMFP. Gravidez na adolescência e outros fatores de risco para mortalidade fetal e infantil no Município do Rio de Janeiro, Brasil. Cad. Saúde Pública. 2010;26(3):567-78.

13. Trajano MFC, Quirino GS, Gonçalves GAA. Consequências da maternidade na adolescência. Cogitare enferm. 2012;17(3):430-6.

14. Dworsky A, Courtney ME. The risk of teenage pregnancy among transitioning foster youth: Implications for extending state care beyond age 18 . Children Youth Serv Rev. 2010;32(10):1351-6.

15. Omar K, Hasim S, Muhammad NA, Jaffar A, Hashim SM, Siraj $\mathrm{HH}$. Adolescent pregnancy outcomes and risk factors in Malaysia. Int J Gynaecol Obstet. 2010;111(3):220-3.

16. Backes MTS, Soares MCF. Poluição ambiental, residência materna e baixo peso ao nascer. Rev. bras. enferm. 2011;64(4):639-50.

17. Ministério da Saúde (BR). Manual Técnico: Pré-natal e puerpério, atenção qualificada e humanizada. $2^{\mathrm{a}}$ ed. Brasília : (DF); 2006.

18. Uchimura TT, Pelissari DM, Uchimura NS. Baixo peso ao nascer e fatores associados. Rev. Gaúcha Enferm. 2008;29(1):33-8.

19. Goldenberg P, Figueiredo MCT, Silva RS. Gravidez na adolescência, pré-natal e resultados perinatais em Montes Claros, Minas Gerais, Brasil. Cad. Saúde Pública. 2005;21(4):1077-86.

20. Awoleke JO. Maternal risk factors for low birth weight babies in Lagos, Nigeria. Arch Gynecol Obstet. 2012;285(1):1-6. 